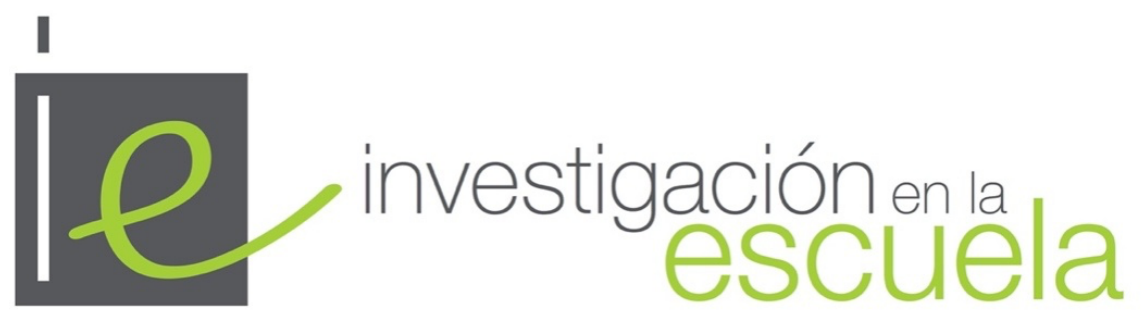

Revista de Investigación e Innovación Educativa nº 103, 2021 | e-ISSN 2443-9991

\title{
¿Podemos cultivar este suelo? Una secuencia didáctica para futuros maestros contextualizada en el huerto
}

\author{
Can we cultivate this soil? A teaching-learning sequence for pre-service teachers contextualized at the garden
}

Dr. Daniel Zuazagoitia es Profesor Adjunto en la Facultad de Educación y Deporte de la Universidad del País

iD Vasco/Euskal Herriko Unibertsitatea (UPV/EHU) (España)daniel.zuazagoitia@ehu.eus ·https://orcid.org/00000001-9954-7855

Dr. Aritz Ruiz González es Profesora Adjunto en la Facultad de Educación y Deporte de la Universidad del País

iD Vasco/Euskal Herriko Unibertsitatea (UPV/EHU) (España) · aritz.ruiz@ehu.eus · https://orcid.org/0000-0001$7409-4634$

iD Dra. Lourdes Aragón es Profesora Contratada Doctor en la Facultad de Educación de la Universidad de Cádiz (España)·lourdes.aragon@uca.es·https://orcid.org/0000-0003-3062-9734

iD Dra. Marcia Eugenio-Gozalbo es Profesora Titular de Universidad en la Facultad de Educación de Soria, Universidad de Valladolid (España)·marcia.eugenio@uva.es·https://orcid.org/0000-0002-7907-9780

Cómo citar este artículo

Zuazagoitia, D., Ruiz-González, A., Aragón, L. y Eugenio-Gozalbo, M. (2021). ¿Podemos cultivar este suelo? Una secuencia didáctica para futuros maestros contextualizada en el huerto. Investigación en la Escuela, 103, 32-47. doi: http://dx.doi.org/10.12795/IE.2021.i103.03

Resumen. En este trabajo se describe el diseño de una Secuencia de Enseñanza-Aprendizaje (SEA) contextualizada en el Huerto Ecodidáctico (HED) y enfocada a mejorar la comprensión del modelo científico de suelo en los maestros de Infantil y Primaria en formación inicial. La SEA presenta una orientación constructivista que combina el enfoque de cambio conceptual y la estrategia de enseñanza-aprendizaje por indagación. Está estructurada en tres fases, y en la central, los estudiantes llevan a cabo un diagnóstico del estado de salud del suelo del HED universitario siguiendo un protocolo de ciencia ciudadana, que se ha integrado para promover la realización de prácticas científicas en el proceso de reconstrucción del modelo mental de suelo. Se apuntala teóricamente el diseño de la secuencia, entre otros sobre el análisis epistemológico y las demandas de aprendizaje, y se presenta un análisis prospectivo ( $\mathrm{n}=54$ ) mediante fenomenografía de las concepciones de suelo inicial y final de los estudiantes. Se observa que: (1) inicialmente, el alumnado presenta modelos estáticos y una visión utilitarista del suelo, (2) la SEA facilita progresos en el aprendizaje, sobre todo en cuanto a comprensión de los componentes del suelo, y (3) la necesidad de incidir en un modelo ecosistémico y de suelo como recurso no renovable. Este estudio refuerza la importancia de emplear contextos reales y situaciones significativas en la enseñanza de las ciencias, tales como los HEDs.

Abstract. In this study, we describe the design of a Teaching-Learning Sequence (TLS) contextualized at an Organic Learning Garden (OLG), and focused on improving the understanding of the scientific model of soil in pre-service teachers of Pre- and Primary School. The TLS follows a constructivist orientation that combines an approach of conceptual change and a strategy of inquiry-based science teaching. It is structured in three phases, and a diagnosis of soils' health is conducted by students by following a protocol of citizen science at the university garden. Such protocol has been integrated into the TLS in order to promote the realization of scientific practices in the process of reconstruction of mental model of soil. Its design is theoretically underpinned on the epistemological analysis and learning demands, among others. A prospective analysis $(n=54)$ of the initial and final soil conceptions held by students is presented. It is observed that: (1) initially, students hold static models and a utilitarian vision of soil, (2) learning 
progresses occur, particularly in terms of understanding soil components, and (3) there is a need to promote an ecosystemic model and a view of soil as a non- renewable resource. This study highlights the importance of using real contexts and significant situations in science teaching, such as OLGs.

\section{Palabras clave $\cdot$ Keywords}

Formación inicial del profesorado, ciencia ciudadana, competencia científica, enseñanza de las ciencias en contexto, huertos ecodidácticos, suelo.

Initial teacher training, citizen science, scientific literacy, Context based science education, organic learning gardens, soil.

\section{Introducción y estado de la cuestión}

El suelo constituye un recurso fundamental, indispensable para la vida en la Tierra. Según el «Informe Técnico sobre el Estado Mundial del Recurso Suelo» realizado por la Organización de las Naciones Unidas para la Alimentación y la Agricultura (FAO) y el Grupo Técnico Intergubernamental Alianza Mundial por el suelo (GTIS) (2015), las presiones humanas sobre él están llegando a límites críticos. La pérdida de suelos productivos puede incrementar de manera significativa los precios de los alimentos y esto, a su vez, aumentar el número de personas que vivan en situación de pobreza y sufran hambre e inseguridad alimentaria, así como otros problemas sociales de gran relevancia: desplazamientos, violencia e injusticia social (ReyesSánchez, 2012). Así pues, el uso sostenible del suelo es uno de los principales desafíos socioambientales al que nos enfrentamos, comparable en importancia y estrechamente relacionado con otros, como el cambio climático y la pérdida de biodiversidad (Fernández et al., 2017).

Según Alcalde (2015), el suelo es un gran desconocido para la sociedad en general y en consecuencia es necesario ponerlo en valor desde las escuelas y a lo largo de todas las etapas educativas. En España, el concepto de suelo se trabaja prácticamente durante toda la enseñanza obligatoria y postobligatoria, de modo que, a su finalización, es esperable que los estudiantes sean capaces de responder a cuestiones biológicas, geológicas, ecológicas y socioeconómicas sobre el suelo (Martínez Peña et al., 2016). Sin embargo, diversos trabajos de investigación educativa constatan la existencia de ciertas deficiencias, asociadas a un tratamiento didáctico inadecuado del concepto de suelo. En Educación Primaria, por ejemplo, Vila Calzado et al. (2017), y en base a un análisis del contenido de libros de texto del $3^{\circ}$ ciclo, señalan errores o simplificaciones que conllevan dificultades para un aprendizaje adecuado. Así, los libros parecen transmitir la idea de suelo como superficie inerte, con una visión utilitarista, de soporte para actividades humanas, y lo definen como la «superficie que pisamos». En las etapas de ESO y Bachillerato, Alcalde (2015) detecta ausencia de contenidos relacionados con la importancia del suelo, como por ejemplo las funciones y servicios que ofrece. Sobre estas dos etapas, la Sociedad Española de la Ciencia del Suelo publicó en 2017 el denominado Libro Blanco, sobre el tratamiento del suelo en los libros de texto de ESO y Bachillerato en España, partiendo de un análisis y ofreciendo sugerencias y recursos didácticos (Gardini, 2017).

A nivel curricular, el concepto de suelo se introduce desde diferentes perspectivas y enfoques, siguiendo las distintas disciplinas científicas básicas que han contribuido a su conocimiento a lo largo de la historia, siendo las más comunes: (1) una «visión geológica», que concibe el suelo como la capa superficial disgregada y de espesor variable que recubre la corteza terrestre procedente de la meteorización física y química de la roca preexistente; (2) una «visión agrobiológica», según la cual el suelo sirve de asiento a la vegetación, y de él depende la agricultura, que es la base de la subsistencia humana; también sustenta la existencia de vida en la Tierra, ya que hace posible el reciclado de la materia en los ecosistemas terrestres; y (3) una «visión ecológica», que concibe el suelo como una interfase, pues está formado por componentes de todas las capas terrestres: atmósfera, hidrosfera, litosfera y biosfera, y es un sistema en equilibrio dinámico con el entorno (Alcalde, 2015, pp. 332-333).

Rebollo et al. (2005) sugieren la existencia de un paralelismo entre las concepciones iniciales del alumnado respecto al suelo y la evolución de las ideas científicas sobre él a lo largo de la historia, diferenciando inicialmente una «etapa estática», que incluye tres visiones: la agrológica -relacionada con la vida vegetal y el cultivo-; la química -que apareció con los métodos analíticos que permitieron relacionar la composición química del suelo y las plantas-; y una geológica -basada en la idea de que el suelo aparece como producto de la desintegración mecánica de la roca madre-. Posteriormente, con el nacimiento de la Edafología como disciplina, se desarrolló una concepción interdisciplinar que incluía aspectos geológicos, químicos, biológicos, climáticos y morfológicos, y estableció la estructuración del suelo por horizontes. Finalmente, se dio un cambio de paradigma al poner el foco en el papel que juegan las comunidades bióticas edáficas, surgiendo una «visión ecológica» del suelo, que lo considera un sistema dinámico, complejo, en el que tienen lugar procesos físicos, químicos y biológicos tendentes al equilibrio con el medio en el que se 
desarrolla. Así, es posible observar que estudiantes de distintas edades y países poseen ideas similares en torno al suelo. Reyes-Sánchez (2012), constata que niños de $5^{\circ}$ y $6^{\circ}$ curso de educación básica en México manejan la idea de suelo como un material que pisamos, y sobre el que se asientan edificaciones, otorgándole un valor puramente utilitario, y detecta confusión entre los conceptos tierra y suelo. Resultados similares se observaron en estudiantes españoles de entre 12 y 17 años, con ideas iniciales coincidentes en cuanto a mostrar dificultades derivadas de la polisemia del término suelo (al referirse la mayoría del alumnado al suelo como la superficie que pisamos), y visiones más acordes con la agrológica y, en menor medida, con la geológica (Yus y Rebollo, 1993). En un estudio más reciente en estudiantes de $4^{\circ}$ de ESO, Fernández et al., 2017), presentan también concepciones en las que predomina el suelo como elemento inerte, y sólo algunos manejan modelos acordes a una visión ecológica, que tiene en consideración el ciclo de la materia, sosteniendo que el origen del suelo está relacionado con la descomposición por parte de organismos. El término «descomposición», según Saéz Bondía et al. (2020), es empleado a modo de comodín por el alumnado, para hacer referencia a aquellos fenómenos que no saben explicar, derivado del uso que hacen de este término en su vida diaria, y vinculándolo a una característica propia de la materia orgánica. Similares ideas previas y dificultades de aprendizaje se han detectado en maestros en formación inicial; Aragón y Cruz (2016) muestran, a partir del análisis de mapas conceptuales, que los futuros maestros de Infantil conciben el suelo como la capa más superficial de la tierra, caracterizada por tener una estructura formada por una mezcla de restos minerales y materia orgánica. En ese estudio, ninguno de los estudiantes mostró una visión dinámica o ecológica del suelo (según Rebollo et al., 2005), sino concepciones más próximas a las visiones agrológica y química.

En consecuencia, son necesarias propuestas didácticas a lo largo de las distintas etapas educativas que estén enfocadas a hacer progresar las ideas de los estudiantes sobre el suelo hacia un modelo más complejo y cercano al propuesto por la ciencia. Estas propuestas deben abordar el suelo desde una visión multidisciplinar en la que se conecten los aspectos básicos de todos los enfoques de la forma más sintética posible y comprensible a distintos niveles (Alcalde, 2015; Reyes-Sánchez, 2012). Brevik y Arnold (2015) inciden en la necesidad de superar viejas definiciones de suelo e incluir nuevas concepciones desde la pedología para los ecosistemas naturales y agrícolas tradicionales, y además también para los entornos urbanos y otros entornos alterados antropogénicamente. Proponen seis elementos clave que la definición de suelo debería tener: (i) los suelos son cuerpos naturales, (ii) los suelos son espaciales y temporales, (iii) los suelos se forman en la superficie, (iv) los suelos son el resultado de complejos procesos biogeoquímicos y procesos físicos, (v) los suelos son capaces de sustentar la vida, y (vi) los suelos pueden cartografiarse a escalas adecuadas. Traducimos aquí la nueva definición de la Sociedad de Ciencias del Suelo de América (SSSA) (Van Es, 2017, p. 2):

$\mathrm{La}(\mathrm{s})$ capa(s) de mineral generalmente suelto y/o materia orgánica que se ven afectadas por procesos físicos, químicos y/o biológicos en o cerca de la superficie terrestre y por lo general contienen líquidos, gases, y biota, y son el soporte para plantas.

\section{Marco teórico}

En el marco del constructivismo, el aprendizaje se conceptualiza como un proceso de reconstrucción de conocimientos que se desarrolla a lo largo del tiempo; los educandos parten de sus ideas o concepciones iniciales, y se pretende que ésas vayan evolucionando de forma progresiva para acercarse a las que propone el educador, es decir, la transposición didáctica del conocimiento científico, al que se denomina «ciencia escolar» (Chevallard, 1991). En este marco se plantea el diseño de «Secuencias Didácticas o Secuencias de Enseñanza-Aprendizaje» (SEAs), productos curriculares de pequeña o mediana escala que cubren la enseñanza y el aprendizaje de un tema científico específico (Guisasola y Oliva, 2020). Diseñar secuencias didácticas supone seleccionar y organizar contenidos en un nivel educativo dado, escoger o diseñar actividades y secuenciarlas, definir los objetivos y prever las dificultades de aprendizaje que puedan aparecer, seleccionar la estrategia metodológica más adecuada, y plantear actividades de evaluación que estén alineadas con los objetivos y la estrategia metodológica escogida (Furió et al., 2020).

El diseño e implementación de SEAs tiene una larga tradición en la enseñanza de las ciencias desde hace más de tres décadas (Psillos y Kariotoglou 2016). Esta tradición se ha enriquecido con la metodología de las investigaciones basadas en el diseño (IBD), que actualmente constituye una importante línea de investigación educativa (Guisasola y Oliva, 2020). Así, el equipo de investigación (los diseñadores) construyen un producto (la SEA) que se implementa y evalúa para detectar problemas y para rediseñar la SEA, en un proceso cíclico de mejora. Su punto fuerte radica en vincular fuertemente la enseñanza y el aprendizaje sobre un determinado tema científico, obteniéndose secuencias cuyas actividades de enseñanza- 
aprendizaje se encuentran adaptadas empíricamente al razonamiento de los estudiantes, en un proceso de investigación intervencionista basado en el diseño (Bell, 2004). Es por tanto imprescindible evaluar los resultados de aprendizaje conseguidos, y conectarlos después con el diseño (Guisasola et al., 2017).

Según Giné y Parcerissa (2003) y Zabala y Arnau (2007), una SEA se estructura en tres fases. La fase inicial o de apertura implica presentar el tema, motivar al alumnado hacia su aprendizaje, e identificar y evaluar los intereses, motivaciones y conocimientos iniciales de los estudiantes, de modo que el docente pueda tomar decisiones en favor de una mayor adaptación de las actividades. La fase de desarrollo está enfocada a la reconstrucción de conocimiento, y durante ella el docente debe ir regulando, es decir, adaptando la secuencia a las necesidades, problemas y situaciones que puedan surgir. La fase final o de cierre debe favorecer que se recapitulen y sinteticen los contenidos trabajados; es importante que incluya actividades de aplicación del conocimiento y también de evaluación final, que en ocasiones pueden coincidir. En el caso de las secuencias, los resultados de aprendizaje pueden medirse como una distancia entre las ideas iniciales y finales de los estudiantes.

Por otra parte, la «competencia científica» es el eje estructurador del currículo de las materias científicas (Jiménez-Aleixandre, 2010) desde la entrada en vigor de la Ley Orgánica de Educación (Ley Orgánica $2 / 2006$, LOE). El enfoque competencial se considera valioso porque permite situar en el centro de la enseñanza la consecución de aprendizajes funcionales y útiles; proponer contextos de aprendizaje más relacionados con las situaciones reales de uso del conocimiento; y promover que la evaluación se centre en comprobar qué sabe hacer el alumno con lo que ha aprendido (Pedrinaci, 2012; Prieto et al., 2012). Para promover la competencia científica del estudiante es necesario implementar metodologías de tipo activo; una de ellas es la «enseñanza de las ciencias en contexto» (Gilbert et al., 2010). Consiste en relacionar la ciencia con la vida de los estudiantes, y hacerles notar el interés que tiene en las dimensiones personal, profesional y social (Caamaño, 2011); es decir, en contextualizar la ciencia que se enseña, o en construir la ciencia escolar a partir de necesidades contextualizadas (Pro y Rodríguez, 2010). Blanco et al. (2012) señalan que la introducción de las competencias en los currículos de la educación obligatoria y también en el programa PISA -que propone situaciones contextualizadas en cinco grandes ámbitos: salud, recursos naturales, medio ambiente, riesgos y fronteras de la ciencia y la tecnología; y en tres niveles de relevancia: personal, social y global- ha puesto de relieve la necesidad de tener en cuenta los contextos tanto en la enseñanza como en la evaluación.

Sin embargo, contextualizar la enseñanza científica es un reto para los docentes; algunas dificultades que se han detectado son la propia identificación de contextos, la definición de su papel en la secuencia, su influencia en la selección de elementos de la competencia científica y la relación entre el contexto y la evaluación (Blanco et al., 2012). En este trabajo, proponemos el uso de Huertos Ecodidácticos (HEDs) como contextos adecuados para promover la competencia científica y el interés por la ciencia de nuestro alumnado. El término HED sincretiza dos conceptos fundamentales: (1) que se trata de huertos cuyo manejo sigue los principios de la agricultura ecológica o la permacultura, y (2) que su principal función no es productiva, sino educativa. Estos dos aspectos caracterizan a este recurso, puesto que hay dos tipos de saberes que son necesarios para el docente que los utiliza: el del manejo de la tierra, y el del uso educativo (Sáenz-Rico, 2017). En base a trabajos anteriores, sabemos que los huertos son contextos de la vida real capaces de activar los conocimientos y experiencias previas de los estudiantes, lo cual facilita la construcción de aprendizajes significativos (Eugenio y Aragón, 2016). Sabemos también que son espacios educativos adecuados en el marco de un modelo de enseñanza-aprendizaje por competencias, puesto que movilizan las dimensiones cognitiva, procedimental, emocional y relacional (Eugenio-Gozalbo et al., 2019). Además, facilitan el tratamiento de temas relevantes en relación a los ámbitos del medio ambiente, la agricultura y alimentación, todos importantes para la sostenibilidad (Eugenio-Gozalbo et al., 2018; 2021). Finalmente, proporcionan oportunidades para construir ideas clave que sean útiles no solo para interpretar las situaciones o resolver el problema o derivado del contexto, sino también otros muchos, lo cual se ha considerado particularmente relevante en la literatura (Marchán Carvajal y Sanmartí, 2015).

\section{Objetivos}

Este trabajo persigue dos objetivos fundamentales:

1. Describir el diseño de una SEA constructivista contextualizada en el HED, enfocada al desarrollo de la competencia científica, y a promover el progreso de las ideas de los estudiantes sobre el suelo hacia la construcción de un modelo más cercano al científico.

2. Comprobar si la SEA impulsa esa progresión de ideas mediante el análisis fenomenográfico de las concepciones iniciales y finales del alumnado sobre el concepto de suelo. 


\section{Metodología}

El diseño de la SEA «نPodemos cultivar en el suelo de nuestro huerto?» se hizo en el contexto de un Proyecto de Innovación Docente (PID) de la Universidad de Valladolid (UVa), «Huertos EcoDidácticos», en el que participan 7 universidades españolas y que, en las últimas convocatorias, ha tenido como objetivo el diseño, la implementación y la evaluación de SEAs contextualizadas en los HEDs y orientadas a mejorar la competencia científica de los maestros en formación inicial. Esta SEA fue diseñada por un grupo de seis docentes del área Didáctica de las Ciencias Experimentales, pertenecientes a cuatro universidades: la Universidad de Cádiz (UCA), la Universidad de Sevilla (US), la propia UVa y la Universidad del País Vasco (UPV/EHU). Cabe destacar ciertas cuestiones clave en su diseño:

- Se diseñó desde una perspectiva constructivista, combinando dos estrategias específicas en Didáctica de las Ciencias: el enfoque de cambio conceptual y la estrategia de enseñanza y aprendizaje por indagación.

- Se contextualizó en los HEDs donde se integra un protocolo del Programa de Conservación de Suelos de Ciencia Ciudadana desarrollado por el Centro de Estudios Ambientales de Vitoria-Gasteiz (CEA, 2021) en la que se emplean las "Tarjetas de Salud de los Ecosistemas Agrícolas" (TSEA).

- Se siguieron las consideraciones en cuanto a estructura y objetivos de Giné y Parcerissa (2003) y Zabala y Arnau (2007).

- Se tuvieron en cuenta algunas pautas que proporciona la metodología de Investigación Basada en el Diseño (Guisasola y Oliva, 2020).

- Se consideraron tres dimensiones fundamentales sobre el suelo: 1) la naturaleza y propiedades del suelo; 2) los servicios ecosistémicos que proporciona, y 3) los indicadores y procedimientos para la evaluación de la calidad del suelo.

- Se planteó como actividad nuclear una práctica científica en el contexto del HED universitario, enfocada a diagnosticar el estado de salud del suelo en base a un protocolo de ciencia ciudadana previamente diseñado.

La implementación piloto de la SEA se realizó durante el segundo semestre del curso 2018-2019. En esta participaron 101 estudiantes de los Grados en Educación Infantil y Primaria de dos universidades diferentes ( $\mathrm{n}=38$ de UCA y $\mathrm{n}=63$ de UPV/EHU). Durante esta implementación, la SEA fue evaluada de forma iterativa a medida que avanzaban las 10 actividades que la vertebran. En particular, se detectaron dificultades conceptuales y de argumentación de los estudiantes en torno al concepto suelo a partir del análisis preliminar de sus ideas previas. Así mismo, hubo necesidades de ajuste de los tiempos empleados en varias actividades, y también ciertas carencias a la hora de abordar la evaluación de la competencia científica, que se había planteado como objetivo de la SEA, aunque este aspecto no se aborda en este trabajo.

Posteriormente, se rediseñó, realizándose diversos cambios: corrección y enfoque de ciertas actividades, reordenamiento de la secuenciación, modificación de formato para una recolección de datos más sistematizada y consideración de nuevos instrumentos para evaluar los aprendizajes, como cuestionarios sobre comprensión o problemas contextualizados para verificar la adquisición de competencia científica. La nueva versión se implementó durante el primer semestre del curso 2019-2020 en las titulaciones del Grado en Educación Infantil y Primaria de 6 Campus Universitarios distintos (UVa-Soria, UPV/EHU-VitoriaGasteiz, UJI-Castellón, UV-Valencia y USAL-Zamora), participando un total de 351 estudiantes, de los cuales $3 / 4$ partes fueron mujeres.

En la secuencia se han utilizado diferentes instrumentos para la evaluación del aprendizaje. Entre ellos, se diseñaron dos cuestionarios, ambos de preguntas abiertas y de tipo test, siendo estas últimas sobre percepción y actitudes hacia el suelo en base al material disponible de organizaciones internacionales (FAO, 2015). En este trabajo se ha analizado prospectivamente (para una parte de la muestra, $n=54$, de la $\mathrm{UPV} / \mathrm{EHU}$ ) una de las preguntas abiertas desde un enfoque fenomenográfico. Se trata de un enfoque de investigación de corte cualitativo que tiene como objetivo investigar y describir la gama completa de formas en que las personas piensan o experimentan un concepto o fenómeno (Marton, 1988; 2015). Partiendo de las diversas concepciones sobre el suelo de los estudiantes, y mediante un proceso iterativo de comparación, discusión y redefinición hasta el consenso por parte de los investigadores, se construye un sistema de categorías jerárquicas de creciente complejidad que clasifica la gama de formas de percibir y comprender el concepto analizado (Anexo 1). En este caso, el grado de acuerdo alcanzado entre dos investigadores con respecto a la asignación de cada definición de suelo a cada categoría estuvo dentro del rango sugerido (coeficiente de confianza Kappa de Cohen, $\mathrm{K}=0,93$ ). Finalmente, se realizó una prueba de rangos con signo de Wilcoxon sobre el número de definiciones asignadas a cada categoría antes y después de la intervención, para detectar cambios estadísticamente significativos. 


\section{Resultados}

\subsection{Descripción de la SEA «¿Podemos cultivar en el suelo de nuestro huerto?»}

Cada una de las tres fases en que se estructura la SEA (según Giné y Parcerissa (2003) y Zabala y Arnau (2007), está apoyada en una o dos preguntas motrices, de forma que el estudiante debe resolver problemas, formular hipótesis y predicciones, razonar en base a evidencias, etc., con objeto de hacer evolucionar sus ideas respecto al suelo. A continuación, se describen las principales actividades de las diferentes fases, también se presentan los objetivos y dificultades de aprendizaje a modo de resumen (Figura 1).

Fase 1: ¿Qué es el suelo para ti?

A1-Actividades para explorar las ideas previas de los estudiantes:

Para empezar, se pretende que los estudiantes expliciten individualmente sus concepciones iniciales en torno al suelo. La actividad A1.A (20 min) plantea la siguiente cuestión: «¿Cómo te imaginas que es el suelo por dentro? ¿Qué hay en él? Haz un dibujo indicando el nombre de cada uno de los elementos que incluyas». La actividad A1.B (35 min) consiste en responder el cuestionario arriba mencionado, con preguntas abiertas y otras de tipo test. En la actividad A1.C (20 min), se propone una autoevaluación de la dimensión conceptual de la competencia científica en base a la rúbrica de Pigrau y Sanmartí (2013), adaptada para el tema del suelo.

A2-Actividades para promover el interés de los estudiantes:

Se introduce el tema de la salud de los suelos agrícolas y, con objeto de despertar el interés de los estudiantes, se contextualiza en el HED universitario. La actividad A2.A (10 min) consiste en la visualización de dos vídeos de divulgación de la FAO sobre el tema. En la actividad A2.B (15 min), el docente plantea preguntas como: «¿Pensáis que se puede cultivar en cualquier tipo de suelo? ¿Qué características debe tener un suelo para que se pueda cultivar?», para fomentar un debate razonado, activando los conocimientos del alumnado. Finalmente, se propone responder a una última pregunta (A1.C, $35 \mathrm{~min}$ ), en grupos y por escrito: «Si quisiéramos saber cuáles son las características del suelo de nuestro huerto para poder cultivarlo... ¿Qué necesitaríamos saber en concreto? y ¿Cómo podríamos averiguarlo?». Mediante esta actividad se pretende que el alumnado detecte sus propias carencias conceptuales y ver si es capaz de predecir qué tipo de procedimientos necesitaría para recoger evidencias, y poder contestar a la pregunta inicial.

Fase 2: ¿Qué características tiene el suelo? ¿Qué servicios ecosistémicos proporciona? ¿Cómo saber si un suelo es cultivable?

A3-Actividades para la reelaboración de ideas:

Se solicita de nuevo un dibujo del suelo, esta vez grupal, en color y en un folio tamaño A3; posteriormente, se plantea a los grupos la siguiente pregunta: «iPensáis que los elementos que habéis dibujado están relacionados de alguna manera? Indicadlo mediante flechas sobre el dibujo del grupo» (20 min). Después se ponen en común las respuestas de los grupos, planteando la tarea de esta forma: «Exponed el dibujo y vuestras ideas al resto de compañeros» $(25 \mathrm{~min})$. Es importante que el docente comente los elementos que los estudiantes han incluido en sus dibujos, si se señalan adecuadamente las interacciones entre elementos, o qué tipos de visiones sobre el suelo aparecen (Rebollo et al., 2005). En la siguiente actividad (A3. B, 20 min), los grupos de estudiantes deben contestar por escrito a estas cuestiones:

1. «¿Qué es ahora para vosotros el suelo? Dad una definición;

2. ¿Ha cambiado algo con respecto a la definición individual que hicisteis al principio de la secuencia? Argumentad la respuesta;

3. En base a los elementos que componen el suelo y lo que se ha visto en la actividad anterior, ¿Qué características creéis que es necesario conocer del suelo de nuestro huerto para saber si podemos cultivarlo? Discutidlas en vuestro grupo, y consensuad nuevas respuestas a ellas» 
Figura 1

Resumen de las fases, objetivos, dificultades de aprendizaje, y actividades de la SEA

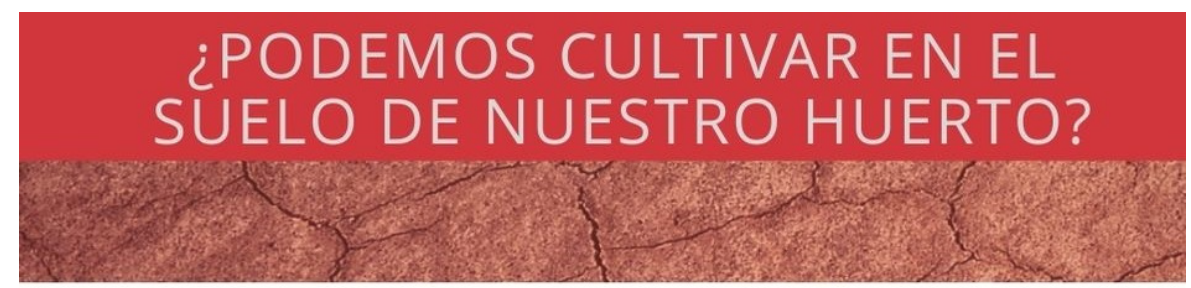

\section{FASE 1 (2H 15') ¿QUÉ ES EL SUELO PARA TI? OBJETIVOS DE \\ 1. Comprender que \\ los conocimientos y el modelo de suelo \\ inicial no son \\ suficientes para \\ abordar la pregunta \\ motriz. \\ DIFICULTADES DEAPENDIZAJE \\ Prevalencia de \\ concepciones del \\ suelo estáticos. \\ Visiones geológicas y \\ agrológicas del suelo \\ (Rebollo et al.,2005: \\ Alcalde, 2015)}

\section{ACTIVIDADES}

A1. $(A, B, C)$ y $A 2$. (A, B, C)

Definiciones y dibujos individuales, debates grupales, autoevaluación de la competencia científica

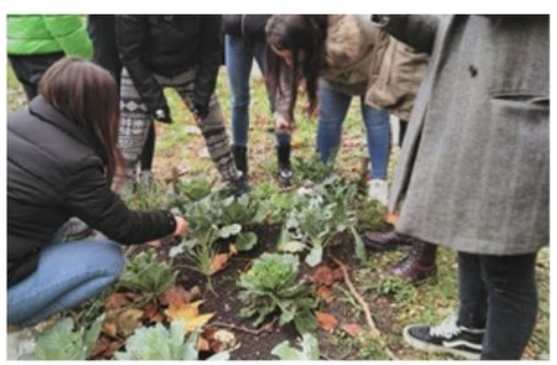

FASE $3\left(3 \mathrm{H}_{0}\right)$ ¿QUEE HE APRENDIDO? ¿CÓMO LO APLICO?

\section{OBJETIVOS DE \\ APRENDIZAJE}

O5. Recapitular el conocimiento adquirido.

O6. Aplicar un mode de suelo equilibrado en sus distintas dimensiones.

07. Argumentar a partir del uso de pruebas frente a un situación problema.

\section{ACTIVIDADES}

A7. (A, B). Realización de un informe grupa sobre el estado de salud del suelo. Respuesta a la pregunta ¿Podemos cultivar en el suelo d nuestro huerto?

A8. (A, B). Aplicación de aprendizajes a través de una situación problema en el contexto del diseño de HED y la salud del suelo

A9. Definición individual de suelo.

A10. Autoevaluación de la competencia científica.

- Dificultades para

sintetizar y discernir relevantes de las que

- Dificultad para evalua sobre la calidad de suelo y generar una fundamentada

-Dificultades en el establecimiento de relaciones causa-efecto

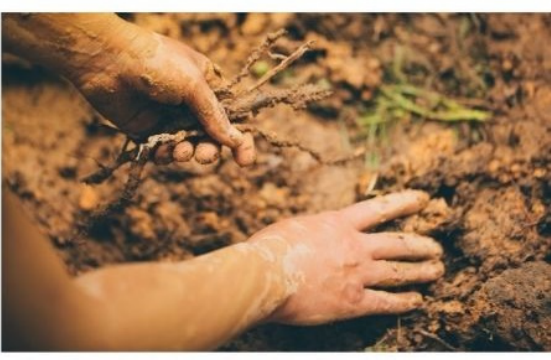

FASE 2 (3H 30') ¿QUÉ

CARACTERISTICAS TIENE EL SUELO? : QUÉ SERVICIOS ECOSISTEMICOS

PROPORCIONA? ¿CÓMO SABER SI UN SUELO ES CULTIVABLE?

\section{OBJETIVOS DE}

\section{APRENDIZAJE}

O2. Comprender e

identificar las

principales

características de

suelo, incluyendo

componentes y

relaciones funcionales entre éstos.

\section{O3. Conocer los}

servicios

ecosistémicos que

proporciona el suelo.

O4. Reconocer qué

parámetros

constituyen los datos y la pruebas

indidad del suelo, y aplicar los procedimientos para conseguir datos sobre ellos.

\section{ACTIVIDADES}

A3. (A, B), A4, A5.

Dibujos y definicione sobre el suelo. Clases magistrales y clases

cooperativas entre

iguales.

Conceptualización de

suelo e indicadores de salud.

A6. (A, B).

Investigación en el

HED a través de las

TSEA. Manipulación y

uso de aparatos para

la toma de medidas

(indicadores) y

diagnóstico del suelo.

Indagación

\section{DIFICULTADES}

-Compresión del

suelo como

ecosistema. En

particular, papel de

os poros, de los

contienen; y la

función dé la biota edáfica

(edafogénesis

descomposición,

existencia de redes

tróficas, nutrición

vegetal)

-Los suelos como reservorio de gestión de los suelos climático.

-Relaciones entre indicadores biológicos y otros indicadores

(químicos, físicos).

-Interpretación del rango de valores de los indicadores propuestos.

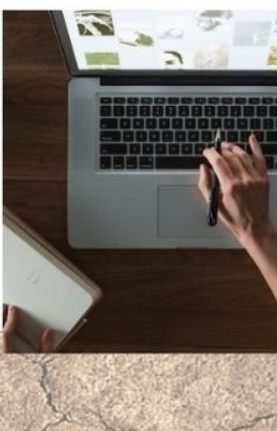
gases y agua que

carbono. Relación existente entre la agrícolas y el cambio 
A4-A5-Mejora del desempeño conceptual:

Estas actividades tienen como objetivo afianzar un concepto de suelo más complejo y cercano al científico-escolar, y promover ciertas destrezas científicas, para saber cómo diagnosticar la salud de un suelo mediante una investigación guiada en el HED universitario. Se comienza con una clase magistral en que se da una visión global de las características del suelo y su salud (composición biótica/abiótica, amenazas, carácter limitado no renovable, indicadores calidad, etc.) y se presenta el Programa de Conservación de Suelos de Ciencia Ciudadana ( $25 \mathrm{~min}$ ). Posteriormente, con el objetivo de que los estudiantes conozcan los indicadores de calidad y sepan cómo realizar el muestreo en el HED, se distribuyen 12 indicadores concretos entre los grupos de trabajo, que corresponden a las 4 dimensiones de los servicios ecosistémicos en que se basan las TSEA («Producción de alimento», «Conservación de la biodiversidad», «Cuidado del suelo», «Mitigación del cambio climático» (Tabla 1). Cada grupo trabaja sobre uno de ellos, documentándose y preparando una presentación que posteriormente expone ante el grupo clase $(50 \mathrm{~min})$. En la página web del programa de ciencia ciudadana se pueden encontrar los materiales necesarios: fichas TSEA, guías para identificación de macroinvertebrados, plagas y malos resultados, así como imágenes de los materiales necesarios para el muestreo y análisis del suelo (CEA, 2021).

\section{Tabla 1}

Servicios ecosistémicos que proporciona el suelo, e indicadores de salud

\begin{tabular}{lcl}
\hline & Dimensiones & \multicolumn{1}{c}{ Indicadores } \\
\hline A & Producción de alimento & 1.- Cosecha y plagas \\
\hline B & Conservación de la & 2.- Diversidad de cultivos. \\
& biodiversidad & 3.- Diversidad vegetal adyacente. \\
& & 4.- Diversidad de macrofauna \\
\hline C & Cuidado del suelo & 5.- Estado biológico (Lombrices). \\
& & 6.- Estado físico (Tiempo de infiltración/circulación del agua). \\
& 7.- Estado físico (Compactación). \\
& 8.- Estado químico (Acidez/basicidad del suelo). \\
& 9.- Estado químico (Uso de Contaminantes/pesticidas). \\
& 10.- Estado químico (Materia orgánica) \\
\hline D & Mitigación del cambio & 11.- Contenido en materia orgánica del suelo. \\
& climático & 12.- Sistema de producción (¿Gana o pierde carbono?) \\
\hline
\end{tabular}

Fuente: Manual de Tarjetas de Salud de Agroecosistemas, Centro de Estudios Ambientales (CEA) de Vitoria-Gasteiz

A6- Investigación en el HED a través de las TSEA:

La actividad central de la SEA es el análisis y diagnóstico del suelo del HED universitario. Previamente, se presentan los materiales a emplear y repasan los procedimientos a seguir para medir cada uno de los 12 indicadores de las fichas TSEA (Figura 2). Se señala que los datos que se obtengan se enviarán al Programa de Suelos de Ciencia Ciudadana, por lo que es importante ser rigurosos en cuanto a seguir los procedimientos establecidos. Cada grupo de estudiantes dispone, para el trabajo en el huerto, de: el protocolo TSEA, el kit de materiales y una ficha para rellenar. El propio programa aconseja llevar a cabo estas tareas en mayo u octubre, por motivos de estado del suelo.

En el HED se escoge un punto de muestreo para cada grupo de trabajo, que debe estar entre líneas de cultivo. En el caso de que haya suficientes grupos de trabajo, es interesante analizar también otros puntos del espacio del HED (fuera de los bancales o zonas de cultivo), para contrastar resultados (1 h $30 \mathrm{~min}$.). Durante esta investigación en el HED, los estudiantes ponen en juego sus conocimientos y su modelo de suelo, usan pruebas para determinar la salud del suelo y, a su vez, discuten y argumentan las posibles relaciones entre los diversos indicadores (compactación, infiltración, materia orgánica, color, numero de lombrices, etc.) (Figura 2). 


\section{Figura 2}

Material básico para la realización del análisis de suelo y extracto de la ficha de campo donde se anotan los datos obtenidos para cada indicador, junto a fotografías de las diferentes fases durante la investigación en el HED

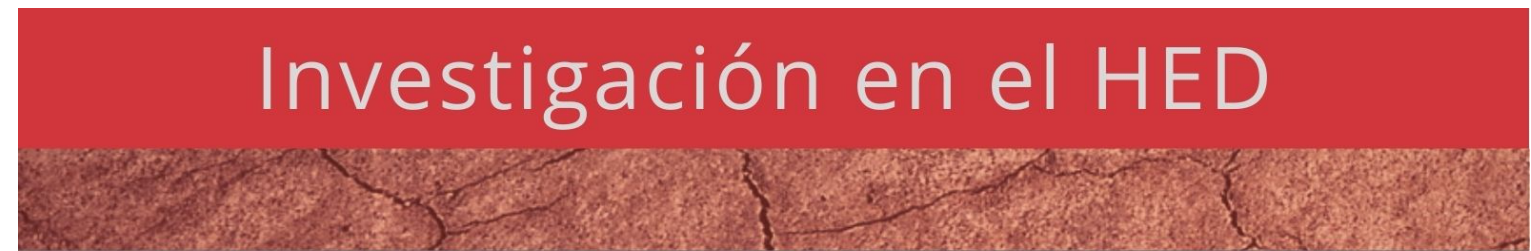

- MATERIALES

1.- Infiltrometro metálico

2.- Maza para clavar el infiltrometro, 3.- Termómetro boli para la temperatura de suelo, 4.- Vaso de precipitadosmultiusos, 5 .Agua oxigenada, 6.- pHmetro o papel tornaso 7.- Frasco lavadorcon agua, 8.- Varilla para la compactación 9.- Pala-azada, 10.- Balanza de mano, 11.- Ficha de campo

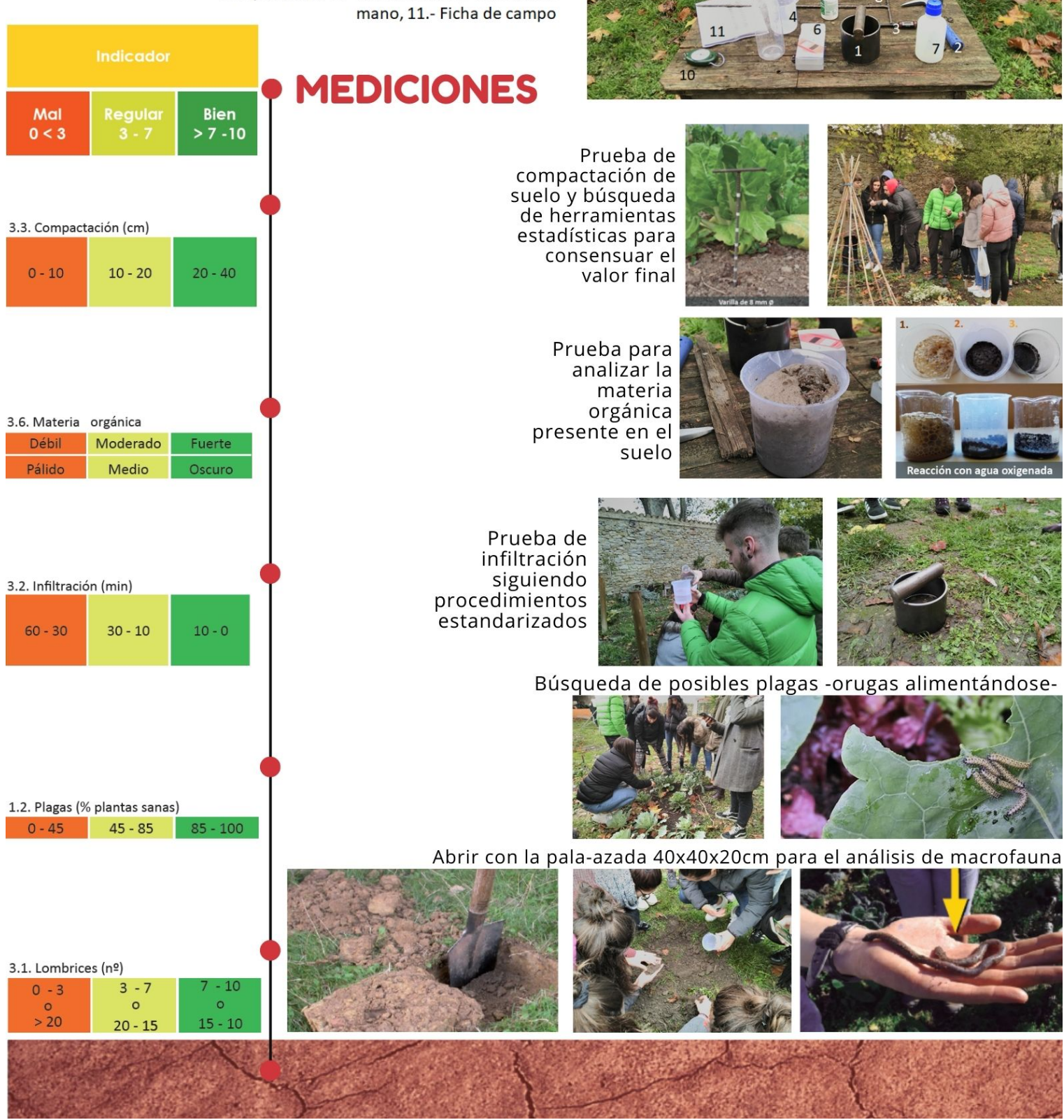


Fase 3: ¿Qué he aprendido? ¿Cómo lo aplico?

De vuelta al aula, se realiza una puesta en común de los resultados obtenidos por los distintos grupos de trabajo en sus puntos de muestreo del espacio del HED, y se comenta y reflexiona sobre las características del suelo, consensuando una valoración general de su adecuación para el cultivo. También se consensuan en una única hoja de campo los valores para cada dimensión; ésta se remite en formato Excel al programa de ciencia ciudadana, para que archiven los resultados obtenidos y puedan realizar un seguimiento de la parcela en los años siguientes.

A7- Elaboración de un informe científico: diagnóstico del suelo en base a las pruebas recabadas:

A modo de conclusión, los grupos de estudiantes preparan un informe sobre el suelo del HED (1h 30 min). Se les proporciona un guion, instándoles a incorporar una serie de puntos evaluables: localización "Google Earth", fotos del HED, descripción visual, resultados y análisis para cada dimensión, tabla de hoja de campo con los datos, explicación de los mismos, limitaciones que posee este estudio a nivel de fiabilidad de los datos, dudas surgidas durante el proceso, manejo de instrumentos, trabajo en equipo, nuevos interrogantes surgidos, conclusiones finales y respuesta a la pregunta nuclear de la secuencia («نPodemos cultivar en el suelo de nuestro huerto? »). Deben argumentar su respuesta en base a las pruebas obtenidas y, finalmente, aportar propuestas para la mejora del suelo, pudiendo consultar en internet.

A8- Aplicación del conocimiento y evaluación en base a la resolución de una situación-problema:

Con el objetivo de aplicar y evaluar algunas dimensiones de la competencia científica, se propone la resolución de un problema contextualizado en un hipotético centro educativo donde se dispone de un terreno que podría utilizarse como huerto escolar. Se aportan varias pruebas (tabla TSEA con datos, texto descriptivo con datos clave sobre la calidad del suelo y una imagen del espacio) y se solicita a los estudiantes, individualmente: «En base a todo lo aprendido a lo largo de la secuencia sobre suelos que hemos desarrollado, comenta los resultados que adjuntamos, de aplicación de las fichas TSEA a un suelo de cultivo, incidiendo en las problemáticas que se detectan y las posibles propuestas de mejora». También se les pide que contesten: «¿Es posible cultivar en el suelo de este huerto?» (30 min).

A9-A10- Evaluación final del concepto suelo (A9) y autoevaluación de la competencia científica (A10):

Para cerrar la SEA, se plantea la misma pregunta abierta que inicialmente: «¿Qué es el suelo?» (15 min). En la actividad A10 (30 min), se propone una autoevaluación de las dimensiones conceptual, metodológica y actitudinal de la competencia científica en base a la rúbrica de Pigrau y Sanmartí (2013), adaptada para el tema del suelo.

\subsection{Análisis fenomenográfico de la evolución del concepto suelo}

Antes de la implementación (pre) y tras la implementación (post) de la SEA, el alumnado ( $\mathrm{n}=54)$ respondió a una pregunta abierta y explicó, de forma individual, qué es el suelo. Mediante un análisis fenomenográfico, se identificaron en las definiciones de los estudiantes tres dimensiones: composición, función y formación. Para cada dimensión, se definió un sistema de 5 categorías, en orden de complejidad creciente (nula, ingenua, básica, intermedia y compleja), que comprendían todo el rango de concepciones (Anexo 1). Tras aplicar la prueba de rangos con signo de Wilcoxon sobre el número de definiciones asignadas en cada categoría antes (pre) y después (post) de la intervención, se detectan cambios estadísticamente significativos $(\mathrm{p}<0,0001)$ en todas, evidenciándose así una progresión significativa en el aprendizaje del concepto de suelo a nivel de grupo (Figura 3).

Inicialmente, los estudiantes mostraron dificultades para definir el suelo (Figura 3, pre). Lo hicieron refiriéndose a aquello que pisamos, una capa formada por una mezcla de cosas y/o elementos indefinidos (p.ej. «capa hecha de diferentes materiales», alumno pre_036). Pocos estudiantes (pre $=5.5 \%$ ) proporcionaron una lista de componentes variados, que responda adecuadamente a la composición de suelo según el conocimiento científico. Así mismo, cuando señalaron alguna de sus funciones (pre=16.6\%), lo hicieron, sobre todo, desde un punto de vista utilitarista ( $p . e j$. «donde vivimos» $\mathrm{O}$ «donde podemos plantar», «donde construimos nuestra vida», pre_028_046_031). Aún más escasos fueron quienes aludieron a la formación o génesis del suelo en la definición (pre=7.4\%), detectándose ópticas diversas ( «Es la capa originada a través de la sedimentación de diferentes materias», «Es una parte superficial de la tierra, la cual se forma por la desintegración de algo físico como las rocas» $\mathrm{y}$ «El suelo es una capa, profunda, formada, a lo largo de todos estos años, por materiales naturales como: tierra, rocas...,etc. ", pre_015 y pre_027, respectivamente). El análisis fenomenográfico constata que, inicialmente, la mayoría del alumnado mostró concepciones situadas en las categorías menos complejas. 


\section{Figura 3}

Categorias extraidas a partir de las definiciones de suelo de los estudiantes, y asignación en \% sobre el total en los momentos pre y post de la SEA para las tres dimensiones consideradas (composición, función y formación)

\section{$\square$ PRE $\square$ POST}

\section{A - Composición del suelo}

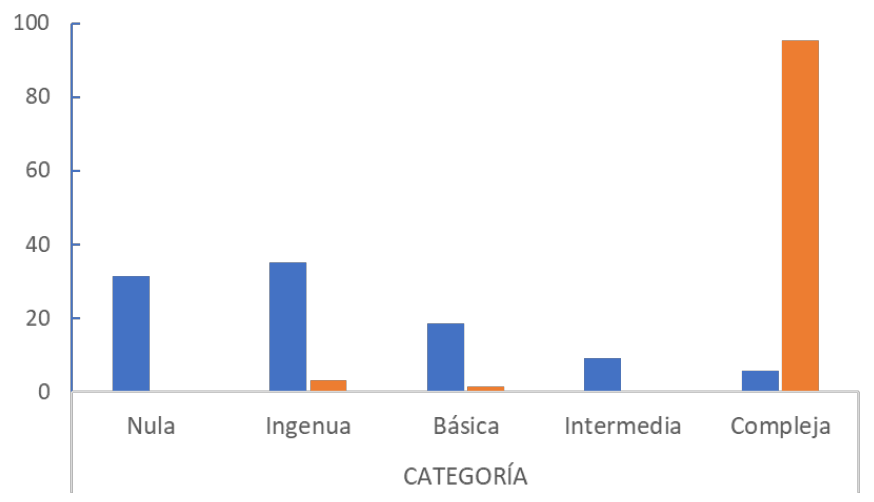

B - Funciones ecosistémicas

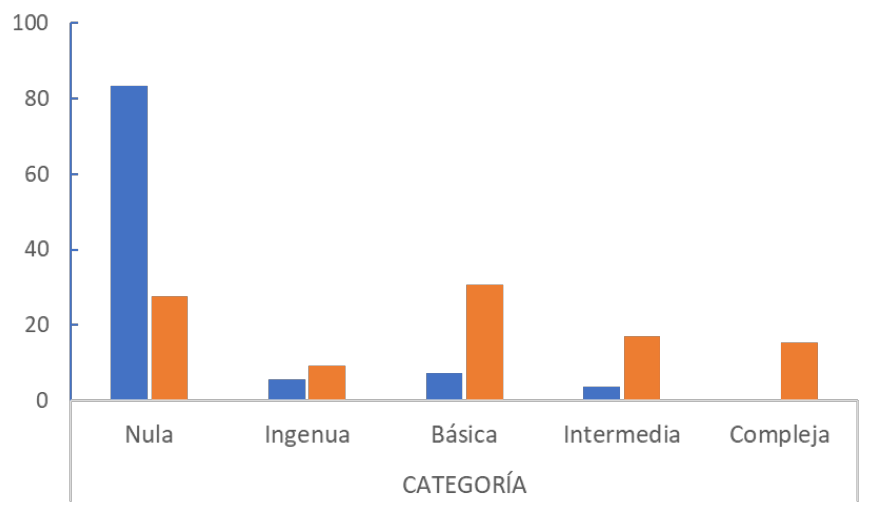

C - Formación del suelo

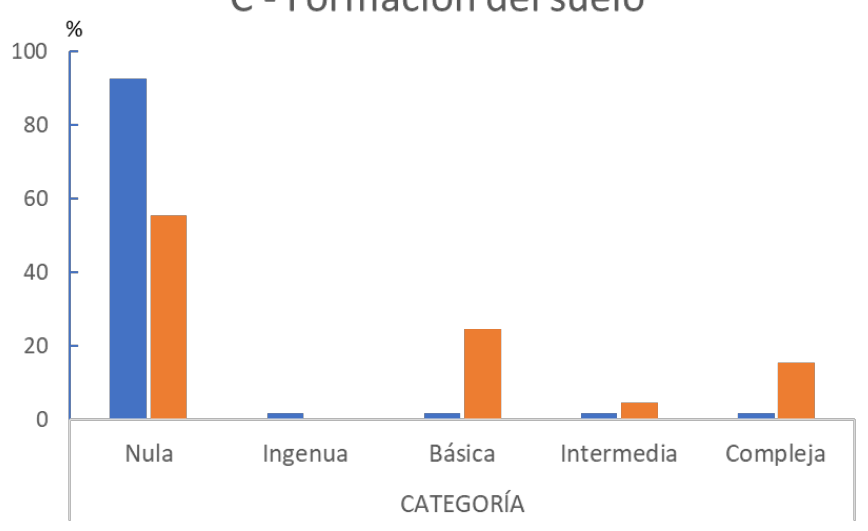

Tras la implementación de la SEA (Figura 3, post), la descripción que hicieron del suelo fue notablemente más larga, descriptiva y presentó conocimientos más concretos $(\not . e j$. «El suelo es la superficie sobre la que vivimos. Está formado por agua, aire, materia orgánica, rocas/minerales y animales. Es la base de la alimentación, ya que casi toda la comida que consumimos procede del suelo. Para su formación se necesitan muchos años, y se ha ido construyendo gracias a la acción del agua y del viento, en su interior se encuentra una gran cantidad de seres vivos -la mayoría no se perciben a simple vista-, que son los encargados de descomponer la materia orgánica y fabricar nutrientes, que luego son utilizados por las plantas», post_063). La composición del suelo fue abordada por todos los estudiantes en sus definiciones, y un 95.3\% lo hicieron de forma adecuada. Las funciones ecosistémicas emergieron prácticamente en las $3 / 4$ partes de 
las respuestas si se consideran las cuatro categorías superiores (pre $=16.6 \%$ frente a post $=72.3 \%$ ), pero mayoritariamente se hicieron desde una aproximación utilitarista o de servicios: el suelo como proveedor de alimentos (p.ej. «El suelo es necesario para la vida de los seres vivos porque de él se obtienen alimentos y también sirve para hacer compost gracias a la materia orgánica que se obtiene en él», post_044). Cabe destacar que, tras la implementación, emergieron la visión ecosistémica y ambiental, que manifestaron prácticamente un tercio de los estudiantes (pre $=3.7 \%$ vs. post $=16.9 \%$ y pre $=0.0 \%$ vs. post $=15,3 \%$, respectivamente), integrando el suelo como agente principal para la vida, y reconociendo su valor y la necesidad de conservarlo. Así mismo, es destacable que hicieron referencia, en muchos de los casos, a su capacidad para descomponer la materia orgánica, y a la importancia de este proceso ( $\not . e j$. «[...]en el suelo se produce el ciclo de vida: las plantas crecen, al morir se convierten en materia orgánica y vuelven a la tierra y se convierten en abono para las plantas que van a comenzar. Podemos encontrar varios niveles, árboles, arbustos y hierba...», post_012). Tras la finalización de la SEA, más de la mitad del alumnado continuó sin hacer referencia alguna a la génesis del suelo (pre $=92.5 \%$ vs. post $=55.3 \%$ ), aunque casi un $30 \%$ mencionó que el suelo se forma mediante procesos de meteorización y descomposición biogeoquímicos, y casi un 15\% señaló además que este proceso es lento y por tanto debemos considerar el suelo como un recurso no renovable, valioso y que debemos conservar $(\not . e j$. «[...] capa superficial del planeta tierra producida por minerales, agua, aire y materia orgánica. Debajo de ella podemos encontrar más compactación y más rocas a medida que la tierra va descendiendo. Un puñado en el suelo podemos encontrar millones de bacterias y para que el suelo crezca un centímetro se necesitan miles de años», post_012). En consecuencia, y aunque se observa una evolución significativa en las tres dimensiones, debería hacerse hincapié en la tercera, la génesis del suelo, y recalcar el valor del suelo como recurso solo renovable a medio-largo plazo.

\section{Conclusiones}

Estamos de acuerdo con Pedrinaci (2012) y Prieto et al. (2012), entre otros, en la conveniencia de enseñar ciencias utilizando contextos o situaciones reales para promover tanto el interés del alumnado, como la consecución de aprendizajes funcionales y útiles. La SEA que describimos en este trabajo integra el uso de los HEDs y de un protocolo de ciencia ciudadana para desarrollar el modelo científico escolar de suelo y la competencia científica de los maestros en formación inicial. En el futuro desarrollo de su profesión, es posible que estos estudiantes se enfrenten a la pregunta-problema nuclear que plantea la SEA («¿Es posible cultivar en este suelo?»), y a la realización de tareas análogas a las que han llevado a cabo durante ella. El HED universitario es, por tanto, el escenario en el que los estudiantes vivencian situaciones reales y las resuelven activamente, aplicando procedimientos científicos, a la vez que desarrollan el interés y la motivación, promovidos en este caso por el hecho de realizar actividades al aire libre, manipulando y experimentando, y conectando teoría y práctica. Defendemos entonces que los huertos son tan necesarios para la educación científica y ambiental en los centros educativos, incluidos los campus universitarios, como lo son los laboratorios. Por otra parte, la integración en la SEA de un protocolo de ciencia ciudadana previamente diseñado ha sido útil en cuanto a ayudar a motivar y dar sentido a la propuesta didáctica. Así mismo, también ha permitido plantear procedimientos científicos confiables y a la vez asequibles al público en general, tal y como se ha observado anteriormente, sobre todo en relación a programas sobre biodiversidad (Bonney et al., 2009; Devictor et al., 2010; Kelemen-Finan et al., 2018).

La SEA que describimos, aunque susceptible de mejora, constituye un producto curricular de calidad, fruto de un proceso de diseño, implementación, evaluación y rediseño, y realizado en colaboración entre docentes de diferentes universidades. Cabe destacar que incluye un análisis epistemológico, establece unos objetivos de aprendizaje acordes con ése y las herramientas para evaluarlos, y enfoca la enseñanzaaprendizaje desde estrategias didácticas que vinculan el conocimiento conceptual con la práctica. De este modo, se desafían las concepciones iniciales de los estudiantes, que les conducen a disonancia cognitiva, promoviendo cambios conceptuales que les permiten interpretar y actuar en la realidad (Duschl et al., 2011). $\mathrm{El}$ análisis exploratorio de datos constata muchas dificultades de aprendizaje que la literatura especializada señalaba (Hayhoe, 2013), sobre todo la visión utilitarista que los estudiantes tienen del suelo, que está relacionado por una parte con el uso cotidiano del término, pero que en parte podría también responder a un paradigma antropocéntrico de interpretación de la realidad (Kopnina, 2011). Se han evidenciado progresos significativos hacia concepciones más complejas y acordes al modelo científico escolar; en concreto, la consideración de las diferentes dimensiones del suelo (composición, función y génesis) mejora, aunque las conexiones establecidas entre ellas son incipientes y en futuras implementaciones debería incidirse en el suelo como recurso no renovable a escala humana, y en sus funciones ecosistémicas. 


\section{Apoyos}

Este trabajo se ha realizado gracias al Proyecto de Innovación Docente (PID) «Huertos EcoDidácticos (HEDs): nuevos espacios para la promoción de la competencia científica de los maestros en formación inicial», de la Universidad de Valladolid (Uva), y también gracias al Proyecto de Sostenibilidad «Realización de prácticas docentes colaborativas e interdisciplinares en el Huerto Ecológico del Campus de Álava», dentro del programa Campus Bizia Lab de la Universidad del País Vasco UPV/EHU, iniciativa impulsada por la Dirección de Sostenibilidad y del Servicio de Asesoramiento Educativo, ambos pertenecientes al Vicerrectorado de Innovación, Compromiso Social y Acción Social. Agradecer igualmente la participación de todas las universidades implicadas en la investigación y a la «Red de Universidades Cultivadas» (RUC), por su apoyo y asesoramiento.

\section{Referencias}

Alcalde, S. (2015). Impulso y difusión de la Ciencia del Suelo en el 2015. Año Internacional de los Suelos (AIS2015). Enseñanza de las Ciencias de la Tierra, 23(3), 330-330. Recuperado de https://bit.ly/3q0jPag

Aragón, L. y Cruz, I. M. (2016). ¿Cómo es el suelo de nuestro buerto? El Aprendizaje Basado en Problemas como estrategia en Educación Ambiental. Una experiencia desde el Grado de Maestro/ a en Educación Infantil. doi: 10.7203/DCES.30.6475

Bell, P. (2004). On the theoretical breadth of design-based research in education. Educ. Psychol, $39,243$. DOI:10.1207/s15326985ep3904_6

Blanco, Á., España, E. y Rodríguez, F. (2012). Contexto y enseñanza de la competencia científica. Alambique, 70, 9-18.

Bonney, R., Cooper, C. B., Dickinson, J., Kelling, S., Phillips, T., Rosenberg, K. V. \& Shirk, J. (2009). Citizen science: a developing tool for expanding science knowledge and scientific literacy. BioScience, 59(11), 977-984. doi: 10.1525 / bio.2009.59.11.9

Brevik, E. C. \& Arnold, R. W. (2015). Is the traditional pedologic definition of soil meaningful in the modern context? Soil Horizons, 56(3), 1-8. https://doi.org/10.2136/sh15-01-0002

Caamaño, A. (2011). Enseñar química mediante la contextualización, la indagación y la modelización. Alambique, 69, 21-34.

CEA. (2021). Centro de Estudios Ambientales: Programas de ciencia ciudadana. [dossier]. Recuperado de http://bit.ly/3pXbgwX

Chevallard, Y. (1991). La transposición didáctica. Del saber sabio al saber enseñado. Editorial AIQUE.

Devictor, V., Whittaker, R. J. \& Beltrame, C. (2010). Beyond scarcity: Citizen science programmes as useful tools for conservation biogeography. Diversity and Distributions, 16, 354-362. https://doi.org/10.1111/j.1472-4642.2009.00615.x

Duschl, R., Maeng, S. \& Sezen, A. (2011). Learning progressions and teaching sequences: A review and analysis. Studies in Science Education, 47(2), 123-182.

Eugenio-Gozalbo, M., Ramos Truchero, G. y Vallés Rapp, C. (2019). Aprendizaje de las ciencias naturales basado en el uso de huertos ecológicos: identificación de las dimensiones percibidas por futuros maestros. Enseñanza de las Ciencias, 37(3), 111-127. https://doi.org/10.5565/rev/ensciencias.2657

Eugenio-Gozalbo, M., Ramos-Truchero, G. \& Suárez-López, R. (2021). University gardens for sustainable citizenship: assessing the impacts of garden-based learning on environmental and food education at Spanish Higher Education. International Journal of Sustainability in Higher Education, 22(3), 516-534. https://doi.org/10.1108/IJSHE-06-2020-0208

Eugenio, M. y Aragón L. (2016). Aproximaciones metodológicas al uso de huertos ecológicos como recurso didáctico y contexto educativo en la formación inicial de maestros/as. Revista Eureka sobre Enseñanza y Divulgación de las Ciencias, 667-679. 13(3), doi: 10.25267/Rev_Eureka_ensen_divulg_cienc.2016.v13.i3.11

Eugenio, M., Zuazagoitia, D. y Ruiz-González, A. (2018). Huertos EcoDidácticos y Educación para la Sostenibilidad. Experiencias educativas para el desarrollo de competencias del profesorado en formación inicial. Revista Eureka sobre Enseñanza y Divulgación de las Ciencias, 15(1), 1501. https://doi.org/10.25267/Rev_Eureka_ensen_divulg_cienc.2018.v15.11.1501

FAO (2015). Año internacional de los suelos. FAO: ¿Cuánto sabes sobre los suelos? [cuestionario] Recuperado de http://bit.ly/2ZYmAhQ

FAO y GTIS (2015). Estado Mundial del Recurso Suelo (EMRS) - Resumen Técnico. Organización de las Naciones Unidas para la Alimentación y Agricultura y Grupo Técnico Intergubernamental del Suelo. Recuperado de https://bit.ly/3aYVSfB

Fernández, A., Sesto, V. y García-Rodeja, I. (2017). Modelos mentales de los estudiantes de secundaria sobre el suelo. Enseñanza de las Ciencias, 35.2, 127-145. http://hdl.handle.net/10347/15482 
Furió, C., Furió, C. y Guisasola, J. (2020). Evaluación de una secuencia de enseñanza de termoquímica para la formación de profesores. Didáctica de las Ciencias Experimentales y Sociales, 38, 133-152. doi: 10.7203/DCES.38.15577

Gardini, A.M. (Coord.). (2017). Libro Blanco sobre el tratamiento del suelo en los libros de texto de Enseñanza secundaria Obligatoria y de Bachillerato en España. Sociedad Española de la Ciencia del Suelo. http://bit.ly/3rczRiI

Gilbert, J.K, Bulte, A.M.W. \& Pilot, A. (2010). Concept development and transfer in context-science education». International Journal of Science Education, 33(6), 817-837. https://doi.org/10.1080/09500693.2010.493185

Giné, N. y Parcerissa, A. (2003). Fases de la secuencia formativa. En N. Giné y A. Parcerisa (Coords.), Planificación y análisis de la práctica educativa (pp. 35-45). Graó.

Guisasola J. y Oliva J.M. (2020). Nueva sección especial de REurEDC sobre investigación basada en el diseño de secuencias de enseñanza. Revista Eureka sobre Enseñanza y Divulgación de las Ciencias, 17(3), 3001. Recuperado de: https://doi.org/10.25267/Rev_Eureka_ensen_divulg_cienc.2020.v17.i3.3001

Guisasola, J., Zuza, C., Ametller, J. \& Gutierrez-Berraondo, J. (2017). Evaluating and redesigning teaching learning sequences at the introductory physics level. Physics Review Physics Education Research, 13, 020139. doi: 10.1103/PhysRevPhysEducRes.13.020139

Hayhoe, D. (2013). Surprising facts about soils, students and teachers! A survey of educational research and resources. In Sustainable agriculture reviews (pp. 1-40). Springer.

Jiménez Aleixandre, M.P. (2010). Competencias en argumentación y uso de pruebas. Graó.

Kelemen-Finan, J., Scheuch, M. \& Winter, S. (2018). Contributions from citizen science to science education: an examination of a biodiversity citizen science project with schools in Central Europe. International Journal of Science Education, 40(17), 2078-2098. https: / / bit.ly/3aXqwpq

Kopnina, H. (2011). Revisiting Education for Sustainable Development (ESD): Examining Anthropocentric Bias Through the Transition of Environmental Education to ESD. Sustainable Development, 22(2), 7383. https://doi.org/10.1002/sd.529

Ley Orgánica 2/2006, de 3 de mayo, de educación (LOE). BOE del 4 de mayo.

Marchán Carvajal, I. y Sanmartí, N. (2015). Criterios para el diseño de unidades didácticas contextualizadas: aplicación al aprendizaje de un modelo teórico para la estructura atómica. Educación Química, 26(4), 267274. Recuperado de https://bit.ly/37OFegw

Martínez Peña, Ma B., Gil Quílez, Maj. y de la Gándara, M. (2016). Aportación de las experiencias a la construcción de modelos: el suelo como sistema. Enseñanza de las Ciencias de la Tierra, 24(2), 182-9. Recuperado de https://bit.ly/3b0Nagw

Marton, F. (1988). Phenomenography: A research approach to investigating different understandings of reality. Qualitative research in education: Focus and methods, 21, 143-161. doi: 10.1177/104973299129121794

Marton, F. (2015). Necessary conditions of learning. Routledge.

Pedrinaci, E. (2012). La noción de competencia científica proporciona criterios para seleccionar, enseñar y evaluar los conocimientos básicos. En E. Pedrinaci (Coord.), 11 ideas clave. El desarrollo de la competencia científica (pp. 15-37). Graó.

Pigrau, T. y Sanmarti, N. (2013). Tresor de recursos: Rúbrica general de la competencia cientifica. [rúbrica]. http:/ / bit.ly/2Mts8xK

Prieto, T., España, E. y Martín, C. (2012). Algunas cuestiones relevantes en la enseñanza de las ciencias desde una perspectiva Ciencia-Tecnología- Sociedad. Revista Eureka sobre Enseñanza y Divulgación de las Ciencias, 9(1), 71-77, 2012. doi: 10498/14625

Pro, A. y Rodríguez, J. (2010). Aprender competencias en una propuesta para la enseñanza de los circuitos eléctricos en la educación primaria. Enseñanza de las Ciencias, 28(3), 385-404. https://bit.ly/3aYV5uT

Psillos D. \& Kariotoglou P. (2016). Iterative desing of teaching-learning sequences. Springer.

Rebollo, M., Prieto, T. y Brero, V. (2005). Aproximación a la historia y epistemología del concepto de suelo: implicaciones didácticas. Enseñanza de las Ciencias, (Extra), 1-5.

Reyes-Sánchez, L.B. (2012). Enseñanza de la ciencia del suelo: estrategia y garantía de futuro. Spanish journal of soil science, 2(1), 87-99. doi: 10.3232/SJSS.2012.V2.N1.07

Sáenz-Rico, B. (2017). S. L. XI Seminario investigación en educación ambiental: avances para la sostenibilidad en la educación superior. Recuperado de https://www.miteco.gob.es/es/ceneam/recursos/documentos/serieea/xi-seminario-investigacioneducacion-ambiental.aspx

Sáez Bondía, M.J., Martínez Peña, Mª.B. y Gil Quílez, Mª.J. (2020). La vida en los suelos. Procesos desconocidos que sustentan los ecosistemas. Alambique: Didáctica de las Ciencias Experimentales, 99, 2834.

Van Es, H. (2017). A new definition of soil. Crops, Soils, Agronomy News, 62(10), 20-21. 
Vila Calzado, V., Vílchez-González, J.M. y Carrillo-Rosúa, J. (2017). Análisis del contenido "suelo" en libros de texto de 3. ${ }^{\circ}$ ciclo de E. Primaria en España. Enseñanza de las ciencias: revista de investigación y experiencias didácticas, (Extra), 497-502. http://bit.ly/3kvb7jb

Yus, R. y Rebollo, M. (1993). Aproximación a los problemas de aprendizaje de la estructura y formación del suelo en el alumnado de 12 a 17 años. Enseñanza de las Ciencias, 11(3), 265-280. https://bit.ly/3qZC4hH

Zabala, A. y Arnau, L. y (2007). Los métodos para la enseñanza de las competencias deben tener un enfoque globalizador. En A. Zabala y L. Arnau (Coords.), 11 Ideas clave. Cómo aprender y enseñar competencias (pp. 163-191). Graó. 
Anexo I. Categorías explicativas definidas para las tres dimensiones principales del concepto suelo (formación, función y composición), y ejemplos de respuestas del alumnado

\begin{tabular}{|c|c|c|c|}
\hline & FORMACIÓN & FUNCIÓN & COMPOSICIÓN \\
\hline NULA & No hace referencia a la génesis & No menciona ninguna función & $\begin{array}{l}\text { No hace referencia a la } \\
\text { composición del suelo }\end{array}$ \\
\hline INGENUA & $\begin{array}{l}\text { Expresa que el suelo siempre } \\
\text { ha estado ahí, manifestando } \\
\text { una visión estática, aunque sea } \\
\text { implícitamente; p.ej.: Llamamos } \\
\text { suelo a lo que está bajo nuestros pies, } \\
\text { puede ser creado por el hombre o por } \\
\text { la naturaleza (027) }\end{array}$ & $\begin{array}{l}\text { Función de soporte } \mathrm{o} \\
\text { superficie; p.ej.: El suelo es donde } \\
\text { vivimos, donde viven plantas o } \\
\text { animales (011) }\end{array}$ & $\begin{array}{l}\text { Visión macro, expresa que el } \\
\text { suelo es una capa, y la } \\
\text { relacionándola con la corteza } \\
\text { superior; p.ej.: El suelo es una } \\
\text { capa fina/delgada de la tierra que } \\
\text { forma una parte esencial de } \\
\text { nuestra existencia para todos los } \\
\text { seres vivos (029) }\end{array}$ \\
\hline BÁSICA & $\begin{array}{l}\text { Hace referencia a algún tipo de } \\
\text { meteorización o ciclo } \\
\text { geoquímico, implicando una } \\
\text { visión dinámica, aunque } \\
\text { parcial. La trasformación a la } \\
\text { que se refiere viene dada por } \\
\text { agentes externos al propio } \\
\text { suelo; p.ej.: Es la superficie de la } \\
\text { corteza terrestre sobre la que se pisa, } \\
\text { y se compone por la desintegración de } \\
\text { las rocas }(015)\end{array}$ & $\begin{array}{l}\text { Plantea } \\
\text { funciones ligadas a la actividad } \\
\text { humana, es una visión } \\
\text { utilitarista; p.ej.: Es una capa } \\
\text { superficial de la tierra la cual los } \\
\text { seres bumanos explotamos para } \\
\text { obtener unos recursos (033) }\end{array}$ & $\begin{array}{l}\text { Expresa que el suelo está } \\
\text { compuesto por varios } \\
\text { elementos, pero no específica } \\
\text { cuales; p.ej.: La suma de } \\
\text { materiales bajo nuestrospies (037) }\end{array}$ \\
\hline INTERMEDIA & $\begin{array}{l}\text { Hace referencia a ciclos } \\
\text { biogeoquímicos, implicando } \\
\text { una visión dinámica. La } \\
\text { transformación a la que se } \\
\text { refiere viene dada tanto por } \\
\text { por agentes externos como } \\
\text { internos; p.ej.: Sedimentación de } \\
\text { tierra, rocas, seres vivos y plantas en } \\
\text { descomposición, etc. en el que se } \\
\text { desarrolla vida (005) }\end{array}$ & $\begin{array}{l}\text { Muestra una visión ecológica } \\
\text { del suelo, interrelaciona } \\
\text { diferentes elementos y aclara } \\
\text { su importancia; p.ej.: El suelo es } \\
\text { una parte fundamental de los } \\
\text { ecosistemas terrestres. Contiene agua } \\
\text { y elementos nutritivos que los seres } \\
\text { vivos utilizan (045) }\end{array}$ & $\begin{array}{l}\text { Expresa que el suelo está } \\
\text { compuesto por varios } \\
\text { elementos, y se centra sobre } \\
\text { todo en los inorgánicos; } p . e j . .: \\
\text { Agua, sales minerales, aire (016) }\end{array}$ \\
\hline COMPLEJA & $\begin{array}{l}\text { Se afirma que el suelo se } \\
\text { forma, es decir, no siempre } \\
\text { estuvo ahí (visión dinámica), y } \\
\text { además esa formación es lenta, } \\
\text { lo que implica que el suelo es } \\
\text { un recurso no renovable a } \\
\text { escala humana; p.ej.: Se ha ido } \\
\text { formando muy lentamente a través } \\
\text { de los siglos por la desintegración de } \\
\text { las rocas superficiales por la acción } \\
\text { del agua, cambios de temperatura, } \\
\text { viento, uso, etc. (004) }\end{array}$ & $\begin{array}{l}\text { Muestra una visión ambiental, } \\
\text { que sitúa también al ser } \\
\text { humano en el ecosistema, en } \\
\text { ocasiones incidiendo en que es } \\
\text { importante y debemos } \\
\text { cuidarlo; p.ej.: Es importante } \\
\text { cuidarlo porque cuando se estropea, } \\
\text { ya no se puede recuperar, no es válido } \\
\text { (051) }\end{array}$ & $\begin{array}{l}\text { Integra las visiones macro y } \\
\text { micro. Capa superior de la } \\
\text { corteza terrestre que está } \\
\text { compuesta por una mezcla } \\
\text { de partículas minerales, } \\
\text { materia orgánica, agua, aire y } \\
\text { organismos vivos; lo que } \\
\text { conforma un medio } \\
\text { ambiente muy complejo y } \\
\text { variable; p.ej.: El suelo está } \\
\text { compuesto por minerales, materia } \\
\text { orgánica, organismos, vegetales, } \\
\text { animales, aire, agua... (026) }\end{array}$ \\
\hline
\end{tabular}

